\title{
Apport d'une étude pluridisciplinaire des débris végétaux dans l'estuaire de la Gironde à la compréhension des phénomènes de colmatage du circuit de refroidissement du Centre Nucléaire de Production d'Électricité du Blayais
}

\section{Contribution of a multidisciplinary study of vegetal debris in the Gironde estuary to understand clogging events of the cooling circuit of the Blayais Nuclear Power Plant}

\author{
A. Fuentes-Cid ${ }^{(1,2)}$, E. De-Oliveira ${ }^{(2)}$, S. Schmidt ${ }^{(3)}$, A. Sottolichio $^{(1)}$, \\ H. Etcheber ${ }^{(3)}$ \\ (1) Univ. de Bordeaux, UMR CNRS 5805 EPOC, 33615 Pessac, France \\ a.fuentes.cid@gmail.com \\ (2) EDF R\&D - LNHE, 6 quai Watier, Chatou, France \\ (3) CNRS, EPOC, UMR 5805, 33615 Pessac, France
}

\begin{abstract}
Résumé - Les débris végétaux sont à l'origine de perturbations d'activités économiques qui nécessitent la filtration de larges volumes d'eau, c'est notamment le cas du Centre Nucléaire de Production d'Électricité du Blayais, implanté sur les rives de la Gironde (Sud-Ouest de la France), où des colmatages du circuit de refroidissement sont occasionnellement observés. Jusqu'à présent, la dynamique des fractions végétales n'avait jamais été étudiée dans ce type d'estuaire macrotidal et hyper-turbide, d'une part, en raison de leur faible quantité, par comparaison à celles des matières en suspension, et d'autre part, du fait de l'absence de protocoles d'étude et d'échantillonnage adéquats. Afin d'améliorer les connaissances englobant ces phénomènes de colmatage, l'objectif de cette étude visait à comprendre la dynamique d'apport et de transit des débris végétaux dans le système fluvio-estuarien de la Gironde. Ceci a impliqué la mise en œuvre d'un suivi spatio-temporel de leur distribution et l'adaptation de techniques (incubations in-situ litter-bag, méthode de prélèvement) pour un estuaire de forte turbidité. Les résultats principaux sont la mise en évidence du contrôle de la distribution des débris végétaux par le régime hydrologique de l'estuaire et la détermination des échelles de temps de leur persistance en Gironde.
\end{abstract}

Mots-clés - estuaire de la Gironde, débris végétaux, matière organique grossière, cinétique de dégradation, litter-bag, flux pulsé, crue, bouchon vaseux 


\begin{abstract}
Vegetal debris have been identified as a factor able to disrupt a wide range of stakeholder activities that require to filter huge volumes of water. The Blayais Nuclear Power Plant, located in the Gironde estuary (S-W France), is subject to occasional blockage intake events by plant debris. Up to now, vegetal fraction dynamic has never been studied in such macrotidal estuaries, due to its small quantity in comparison to the high charge of suspended particulate matter, and due to the lack of appropriate sampling methods. To improve knowledge on the clogging, the objective of this work was to understand the dynamics of vegetal debris input in the fluvio-estuarine system of the Gironde. This work had implied a spatio-temporal survey of their distribution and the development of new techniques (in situlitter-bag incubations, sampling method) adapted to a hyper-turbid estuary. Main results highlight the influence of the hydrological regime on the distribution of vegetal debris and reveal time scales of their persistence in the Gironde Estuary.
\end{abstract}

Key words - gironde estuary, plant debris, coarse organic matter, turnover rate, litter-bag, flood pulse, turbidity maximum zone

\section{INTRODUCTION}

\subsection{Problématique et contexte}

Bien que l'industrie nucléaire mondiale ait reconnu l'importance des événements de colmatage des organes de filtration du circuit de refroidissement des Centres Nucléaires de Production d'Électricité (CNPE) et ait pris des mesures pour les prévenir, la répétition de leur occurrence conduit à porter une attention plus soutenue à leurs causes (WANO, 2008), afin d'être convenablement préparé à prévenir ou à résoudre des événements souvent prévisibles ou des conditions à l'origine d'obstructions.

En France, où presque $80 \%$ de l'énergie produite provient de l'industrie nucléaire, le fonctionnement de plusieurs centrales situées tant en bord de mer (ex. CNPE de Paluel) qu'en estuaire (ex. CNPE de Blayais) ou dans de grands fleuves (ex. CNPE de Cruas ou de Fessenheim ) a été significativement perturbé par des arrivées massives de matériel végétal au niveau des filtres rotatifs de la source froide (Issa, 2010). Alors qu'à Paluel l'origine de ces végétaux est marine (algues), les agents responsables des colmatages dans les autres CNPE indiquent une provenance continentale, soit terrestre (herbes et morceaux de bois au CNPE de Fessenheim), soit aquatique (macrophytes au CNPE de Cruas).

La localisation du CNPE du Blayais, situé sur la rive droite de la partie centrale de l'estuaire de la Gironde, à l'interface des eaux douces et des eaux salées, lui confère un caractère spécifique (Fig. 1). Depuis plusieurs années, son fonctionnement est régulièrement perturbé par l'arrivée massive de débris végétaux (Travade, 2002 ; Travade and Guerin, 2009) sur les 8 tambours rotatifs (maille $3 \times 3$ $\mathrm{mm}$; Fig. 2) qui assurent le système de filtration du circuit de refroidissement avec un débit filtré de $20 \mathrm{~m}^{3} . \mathrm{s}^{-1}$ et sa vitesse de rotation est asservie à une 


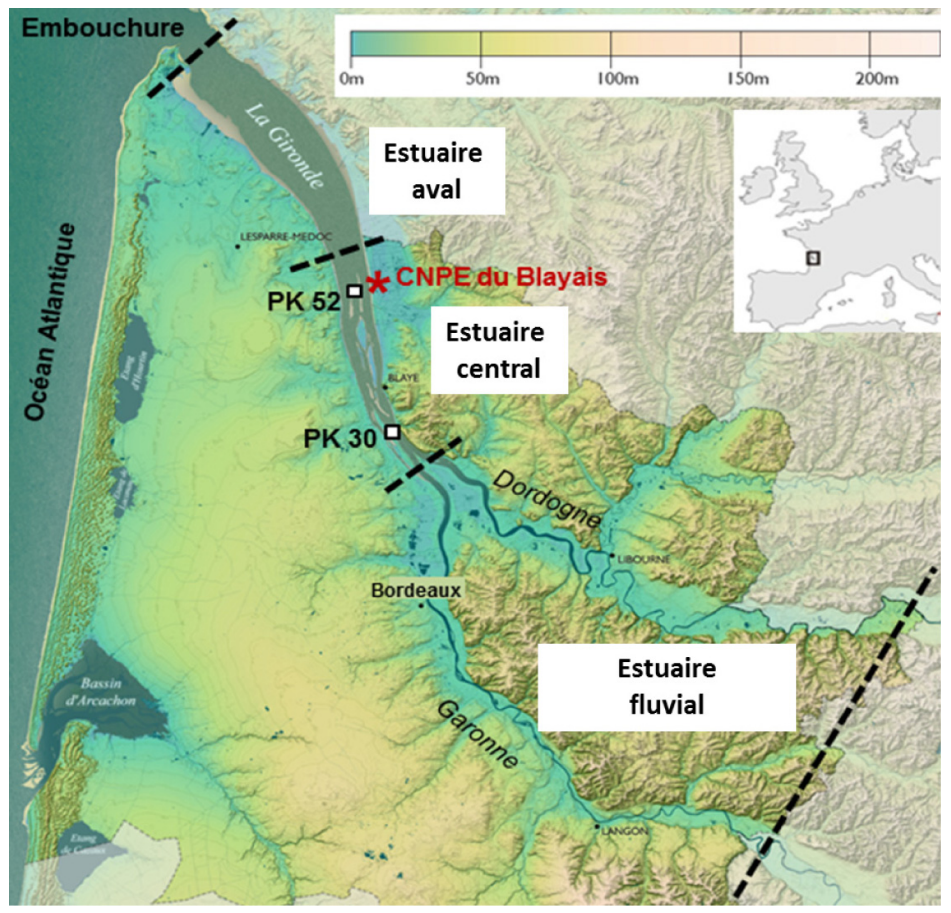

Fig. 1. Carte des reliefs de l'estuaire de la Gironde (extrait et modifié de www.atlas-paysages.gironde.fr) et localisation du CNPE du Blayais. Le point kilométrique 0 (PK 0) est défini à Bordeaux. Les traits en pointillés indiquent le découpage de l'estuaire en : estuaire fluvial, central et aval.

Fig. 1. Relief map of the Gironde estuary (modified from www. atlas-paysages.gironde.fr) and localization of the Blayais NPP. The kilometric point 0 (PK 0) is defined at Bordeaux. Dotted lines represent limits between estuarine sections.



(a)

(b)

Fig. 2. Schéma de fonctionnement d'un tambour rotatif (a) et photographie de sa surface filtrante (maille de $3 \times 3 \mathrm{~mm})(\mathrm{b})$.

Fig. 2. Functioning schema of a rotating drum (a) and picture of its filtering surface ( $3 \times 3 \mathrm{~mm}$ mesh) (b). 


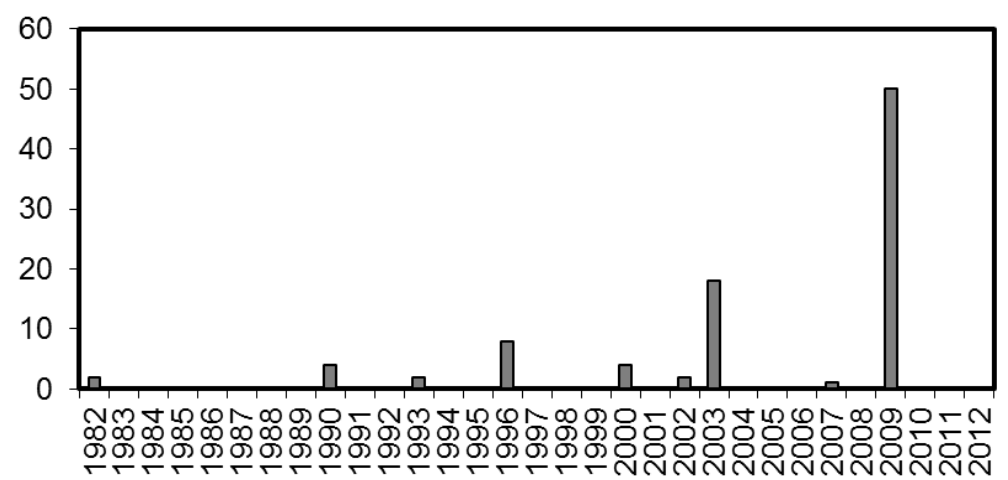

Fig. 3. Nombre d'évènements de colmatage (EVC : arrêt de pompe) par an entre 1982-2012 dans le CNPE du Blayais.

Fig. 3. Number of blockage events per year from 1982 to 2012 in the Blayais Nuclear Power Plant.

mesure de la perte de charge (entre l'amont et l'aval du tambour).

Chaque tambour filtrant est constitué d'un tambour de grande taille (diamètre de $15 \mathrm{~m}$ et d'une largeur de $6 \mathrm{~m}$ ) à axe horizontal dont la périphérie est constituée de panneaux sur lesquels est tendue une toile métallique à maille carrée de $3 \mathrm{~mm}$ de vide de maille. Le tambour est en rotation continue et son nettoyage est assuré, en partie émergée, par des jets d'eau sous pression provenant de l'intérieur du tambour, qui décollent les détritus et les projettent dans une goulotte, les évacuant dans l'estuaire. Pour assurer un nettoyage adapté au degré de colmatage du tambour, ce dernier est pourvu de trois vitesses de rotation qui sont enclenchées en fonction de la perte de charge mesurée de part et d'autre du filtre. Deux vitesses de rotation (« moyenne et grande vitesse », MV et GV respectivement), supérieures à celle dite normale (« petite vitesse », PV), peuvent être enclenchées automatiquement, afin d'améliorer ce processus de filtration. Un épisode de colmatage significatif des tambours correspond donc à la mise en rotation du tambour en GV et, le cas le plus aigu, à l'arrêt de la pompe.

L'intensité de ces colmatages varie significativement selon les années (Fig. 3), déterminant le degré d'impact sur les pertes de productivité et sur les coûts des protocoles nécessaires pour la remise en fonctionnement du processus de production énergétique. En février-mars de l'année 2009, les colmatages des tambours filtrants par des débris végétaux ont entraîné plusieurs arrêts de pompes et une indisponibilité des tranches de durée significative.

Dans le monde, il n'existe pas de références sur l'existence d'un CNPE situé dans un système estuarien ayant subi les débris végétaux comme agent colmatant. Néanmoins, au-delà de leur intérêt scientifique, les débris végétaux ont déjà été reconnus comme des facteurs capables d'affecter de nombreux secteurs d'activités économiques qui impliquent la filtration de grands volumes d'eau : la pêche, les stations d'irrigation, les ouvrages de production d'énergie 
hydraulique et nucléaire (Batalla \& Vericat, 2009 ; Palau et al., 2004). Les débris végétaux (Gómez et al., 2013) menacent occasionnellement les infrastructures, réduisent la productivité des centres de production d'énergie et des systèmes de pompage, et augmentent le coût des opérations.

Le système fluvio-estuarien de la Gironde est représentatif d'un grand nombre d'estuaires européens de type macrotidal, constituant un axe de développement de l'activité humaine en même temps qu'une fonction de réceptacle de toutes les influences de son bassin versant. Jusqu'à présent, la dynamique des fractions végétales dans ses eaux hyperturbides n'y avait jamais été étudiée, en raison de leur faible quantité, par rapport aux fortes charges en matières en suspension fines (plus de 1 g.L ${ }^{-1}$ en surface, Sottolichio et al., 2011). En effet, la forte turbidité des eaux empêche le développement d'organismes photosynthétiques (algues, macrophytes, etc. (Irigoien \& Castel, 1997)) et la contribution de matière organique particulaire autochtone (phytoplancton, macrophytes, etc.) est négligeable en comparaison avec la fraction organique allochtone (Etcheber et al., 2007 ; Savoye et al., 2012). Ceci explique aussi l'absence de protocoles d'étude et d'échantillonnage adéquats de ce matériel végétal.

Dans l'estuaire de la Gironde, considéré comme un des plus grands d'Europe, la limite de la marée dynamique se localise à environ $170 \mathrm{~km}$ de l'embouchure (Fig. 1), dans les sections fluviales de ses deux affluents principaux : la Garonne et la Dordogne. Les fluctuations des débits fluviaux de ces deux cours d'eau sont fortement corrélées avec le déplacement du bouchon vaseux (appelé «Zone de Turbidité Maximale », TMZ) selon l'axe longitudinal de l'estuaire (Etcheber et al., 2011) : en étiage, le bouchon vaseux remonte vers l'amont de l'estuaire sous l'effet prépondérant de l'onde de marée (Sottolichio et al., 2011) ; en crue, il descend vers l'aval de l'estuaire, quand le front de salinité est repoussé par les forts débits d'eau douce.

La tendance actuelle à la baisse des volumes d'eau douce observée dans le continuum fluvio-estuarien (Etcheber et al., 2013) contribue à deux phénomènes récents : une salinisation du système (David et al., 2005) et une remontée progressive du bouchon vaseux vers les sections fluviales (Sottolichio et al., 2011). Ces changements, associés aux caractéristiques du milieu, peuvent entraîner des conséquences sur le processus de dégradation et de transport du matériel végétal transitant dans l'estuaire.

\subsection{Objectifs de l'étude}

Le but de cette étude est, en premier lieu, l'élargissement des connaissances sur la nature et l'origine des débris végétaux de l'estuaire de la Gironde, la compréhension de leur provenance, de leur apparition, de leur transport et de leur comportement dans les eaux estuariennes à l'échelle du bassin versant de la Gironde, tout en intégrant le rôle joué par les modifications ou les tendances environnementales auxquelles cet estuaire est progressivement soumis. En second lieu, il s'agit de cerner les facteurs majeurs influant sur les phénomènes 
Tableau I. Synthèse de la stratégie générale de l'étude.

Table I. Summary of the study general strategy.

\begin{tabular}{|c|c|c|}
\hline Objectif & Zone & Méthode \\
\hline \multirow{2}{*}{$\begin{array}{l}\text { Provenance et identification des } \\
\text { espèces feuillues dans les } 2 \text { fleuves } \\
\text { (Garonne/Dordogne) }\end{array}$} & \multirow{2}{*}{$\begin{array}{l}\text { Bassins versant } \\
\text { amont }\end{array}$} & Étude sur cartes \\
\hline & & Prospection et carottages \\
\hline \multirow{2}{*}{$\begin{array}{l}\text { Processus de décomposition du } \\
\text { matériel végétal en milieu estuarien }\end{array}$} & \multirow{2}{*}{$\begin{array}{l}\text { Zone } \\
\text { fluvio-estuarienne }\end{array}$} & $\begin{array}{c}\text { Dégradation in-situ: } \\
\text { « litter-bag » }\end{array}$ \\
\hline & & Carottages \\
\hline \multirow{2}{*}{$\begin{array}{c}\text { Présence et état physico-chimique de } \\
\text { la fraction végétale }\end{array}$} & \multirow{2}{*}{ Estuaire central } & $\begin{array}{c}\text { Suivi mensuel } \\
\text { de la fraction végétale }\end{array}$ \\
\hline & & $\begin{array}{c}\text { Caractérisation } \\
\text { (physique et biogéochimique) }\end{array}$ \\
\hline $\begin{array}{l}\text { Étude des conditions favorables } \\
\text { à l'arrivée des débris végétaux }\end{array}$ & $\begin{array}{l}\text { Zone autour } \\
\text { des prises d'eau du } \\
\text { CNPE du Blayais }\end{array}$ & $\begin{array}{c}\text { Mise en relation de la base de } \\
\text { données enregistrant les } \\
\text { colmatages, avec : } \\
\text { - mesures au CNPE, } \\
\text { - mesures à l'extérieur du } \\
\text { CNPE, } \\
\text { - conditions hydro-météo. }\end{array}$ \\
\hline
\end{tabular}

de colmatage des systèmes de filtration du circuit de refroidissement du CNPE du Blayais, afin de prévoir les périodes à risque de colmatages et d'envisager des solutions curatives, voire préventives.

Plus particulièrement, ce travail vise à :

- déterminer les caractéristiques physiques et biogéochimiques de la fraction végétale présente dans l'estuaire ;

- estimer la distribution de la fraction végétale dans l'estuaire de la Gironde ;

- circonscrire la provenance des sources/apports majoritaires de ces débris végétaux et déterminer leur cyclicité et les paramètres conditionnant cette variabilité ;

- comprendre la trajectoire parcourue par les débris végétaux et la temporalité de leur déplacement depuis les zones sources jusqu'à l'estuaire central ;

- évaluer le degré de persistance temporelle de ce matériel végétal face aux conditions environnementales estuariennes ;

- améliorer la compréhension des causes induisant les mécanismes de colmatage perturbant le fonctionnement du CNPE du Blayais.

\subsection{Méthode}

Du fait de la complexité du questionnement, de l'échelle spatiale considérée (Garonne, Dordogne et estuaire de la Gironde) et des objectifs affichés, l'approche méthodologique choisie imposait des démarches très distinctes d'une aire d'étude à l'autre (Tab. I). 
Dans un premier temps, la caractérisation physique et biogéochimique des débris végétaux conduit la discussion sur leur provenance préférentielle, laquelle se base fondamentalement sur l'étude des cartes d'occupation de sol et sur l'étude des débits fluviaux comme vecteur d'apport et de transport (Fuentes-Cid, 2014). Puis, concernant l'évolution de la qualité ou le temps de persistance du matériel végétal dans ce système, l'approche classique « litter-bag ${ }^{1}$ » (Gessner et al., 1999; Gessner \& Chauvet, 2002), jamais utilisée jusqu'alors dans des estuaires macrotidaux et hyper-turbides comme le système fluvio-estuarien de la Gironde, confère une originalité particulière aux résultats enregistrés (Fuentes-Cid et al., 2013). Ensuite, l'interprétation et l'élucidation du processus de transport de matériel végétal en Gironde s'appuie sur un suivi mensuel de la fraction végétale par filet à plancton dans la période 2011-2013 (Fuentes-Cid et al., 2014). Complémentairement, l'explication du mécanisme d'interaction des débris végétaux transitant dans les eaux estuariennes avec les installations du CNPE du Blayais est possible grâce au lien qui ressort entre les concentrations de la fraction végétale dans les eaux de lavage des tambours filtrants du CNPE et la base de données enregistrant les phénomènes de colmatage lors de la même période.

(1) Cette méthode consiste à confiner des quantités connues de litière dans des sacs de nylon pour suivre leur décomposition en milieu naturel : la perte de poids au cours de la durée de l'expérimentation est considérée comme un indicateur de ce processus.
Finalement, la mise en perspective globale des résultats obtenus dans ces études conduit à formuler un mécanisme de transport des débris végétaux dans l'estuaire de la Gironde, capable d'expliquer les phénomènes de colmatage au CNPE du Blayais.

\section{DYNAMIQUE DU SAR DANS L'ESTUAIRE DE LA GIRONDE}

\subsection{Nature et origine}

\subsubsection{Définition du Sar}

La fraction végétale transitant dans les eaux estuariennes est constituée par trois groupes majeurs de végétaux terrestres : feuilles, chaumes de graminées et racines. Cependant, l'élément le plus perturbant vis-à-vis du CNPE est le Sar. Ce terme est utilisé localement pour designer des débris de matériel foliaire, de couleur noire, en état de décomposition avancée et de taille réduite $3-10 \mathrm{~mm}$ (Fig. 4). Plusieurs espèces de plantes dicotylédones, caducifoliés et marcescentes, ont été identifiées comme susceptibles de devenir Sar : peuplier (Populus sp.), chêne (Quercus sp.), aulne (Alnus sp.), saule (Salix sp.) sont les espèces majeures.

Les premières indications sur la nature terrestre du Sar ont été évoquées dans des travaux menés entre 2003 et 2010 (Girardin et al., 2004, 2006 ; Guibert et al., 2009 ; Guibert \& Lebarh, 2008 ; Travade \& Guerin, 2009), qui suggéraient une origine fondamentalement naturelle (sans influence d'aucune activité anthropique), principalement par le fait que sa présence au 


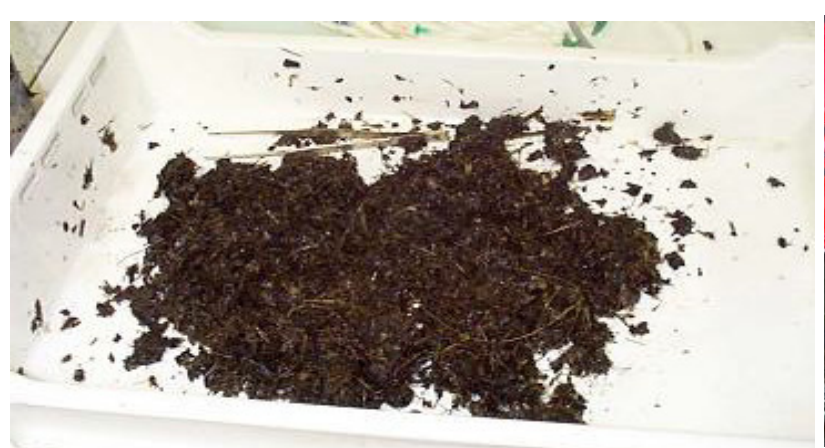

(a)

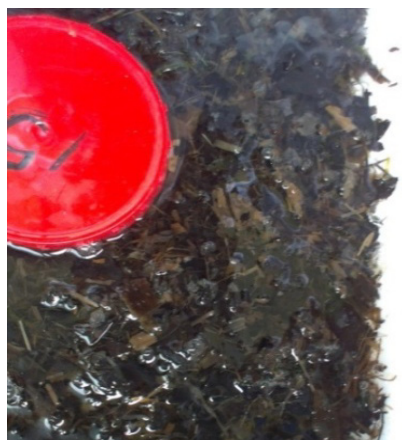

(b)

Fig. 4. Aspect " à sec " (a) et "en suspension dans l'eau » (b) de débris végétaux, riches en Sar, prélevés en 2003 (Lepage et al., 2003) et en 2013 (Fuentes-Cid, 2014).

Fig. 4. "Dried" (a) and "suspended in water" (b) appearance of vegetal debris, highly composed by Sar, sampled in 2003 (Lepage et al., 2003) and in 2013 (Fuentes-Cid, 2014).

niveau estuarien puisse être corrélée aux conditions hydro-climatologiques des saisons automnales et hivernales.

\subsubsection{Caractérisation : physique et biogéochimique}

Les caractéristiques physiques des débris végétaux permettent l'existence de cycles alternant dépôts et remises en suspension dans le milieu estuarien (Fuentes-Cid, 2014). En effet, près des étales de marée, les vitesses de chute sont suffisamment élevées pour provoquer le processus de décantation des débris végétaux vers le fond et leur enfouissement dans la crème de vase, qui se dépose dans ces conditions. À l'inverse, dès que les courants de marée augmentent au cours du cycle de marée, de 0,2 à $2 \mathrm{~m}^{-\mathrm{s}^{-1}}$ (vitesses maximales à mimarée), les débris végétaux sont soumis à des vitesses de courant suffisantes pour induire leur mobilisation et leur remise en suspension dans la colonne d'eau.
Leur caractérisation biogéochimique conduit par ailleurs à vérifier la nature foliaire et arboricole du Sar, et à évaluer son état de dégradation, assez avancé de manière générale, à travers deux paramètres, respectivement, la composition isotopique en carbone et le rapport C:N (Fuentes-Cid, 2014).

En mars 2013, le CNPE a connu des arrivées de Sar sur les tambours de filtration. Des prélèvements dans les eaux estuariennes à cette même période ont montré que le matériel végétal prélevé était plus frais que lors des mois précédents (Fig. 5). $\mathrm{Ce}$ résultat semble confirmer que le matériel biologique présent lors des épisodes de colmatage est transporté plus rapidement vers l'aval (il a passé moins de temps dans l'eau) que lors des périodes sans colmatage.

\subsubsection{Zones/sources émettrices potentielles}

Les affluents de l'estuaire à l'aval de la confluence de la Garonne avec la 


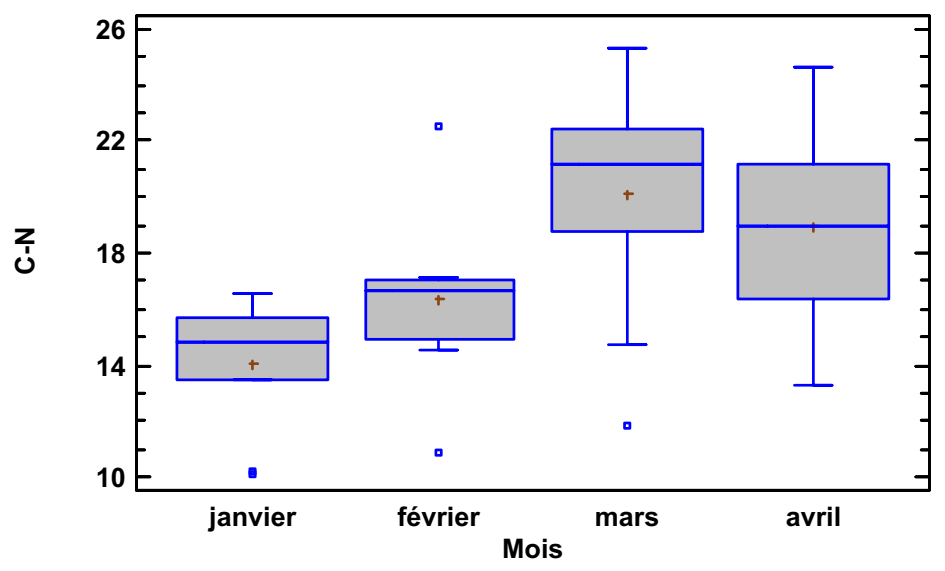

Fig. 5. Rapport C:N des prélèvements des débris végétaux dans l'estuaire en fonction du temps (janvier-avril 2013). Plus la valeur de ce paramètre est élevée, plus le matériel végétal est frais.

Fig. 5. C:N ratio of vegetal debris from the estuary, according to the time (January-April, 2013). The higher the $\mathrm{C}: \mathrm{N}$ value, the fresher the vegetal matter.

Dordogne et jusqu'à l'embouchure océanique drainent $2297 \mathrm{~km}^{2}$ de bassin versant superficiel, ce qui ne représente guère plus de $3 \%$ par rapport à l'ensemble du bassin versant de l'estuaire de la Gironde. Du fait de sa surface très réduite, des débits très faibles des cours d'eau qui le traversent et de la couverture forestière feuillue très parcellaire, la contribution de détritus feuillus issus du bassin versant de la Gironde sensu stricto doit être considérée comme très négligeable.

En revanche, les ripisylves et bois riverains du système GaronneDordogne se caractérisent par une forte production de litière $(400$ à $600 \mathrm{~g} \cdot \mathrm{m}^{-2} \cdot \mathrm{an}^{-1}$ de matériel végétal). Les processus de rétention dans le fleuve et/ou le transfert vers l'aval de ces apports de matière organique allochtone sont déterminés essentiellement par l'intensité des débits fluviaux (Abelho, 2001). II n'y a pas de prépondérance de l'un des deux bassins, Garonne (56 $075 \mathrm{~km}^{2}$ ) ou Dordogne (23 $902 \mathrm{~km}^{2}$ ), en termes de quantités de matières végétales amenées à l'estuaire.

Du fait des réseaux de barrages présents dans ces deux fleuves, principalement en Dordogne, la majorité des apports végétaux devraient parvenir à l'estuaire depuis la Garonne moyenne (aval de la retenue de Malause) et depuis la basse Dordogne (aval du barrage d'Argentat). Ceci correspond aux sections caractérisées par des boisements fluviaux denses, diversifiés et d'épaisseur variable, qui ont une susceptibilité particulière aux montées des eaux lors des crues.

\subsection{Formation du Sar}

Les apports foliaires ripariens ou des couvertures forestières des bassins versants, susceptibles d'entrer 
dans le système estuarien, englobent une variété de caractéristiques structurales. Ces dernières, en plus des paramètres biotiques et abiotiques liés au milieu, influencent le temps de transformation vers leur produit de dégradation.

L'étude de la cinétique de dégradation des feuilles, basée sur la technique litter-bag (Chauvet, 1987 ; Gessner \& Chauvet, 2002 ; Gessner et al., 1999 ; Lecerf et al., 2007 ; Lopes et al., 2011 ; Mateo \& Romero, 1996 ; Montemayor et al., 2011 ; Quintino et al., 2009 ; Sangiorgio et al., 2008) a été conduite pour la première fois dans un estuaire macrotidal hyper-turbide (Fuentes-Cid et al., 2013) en vue de comprendre comment le matériel foliaire transféré des bassins versants réagit dans un environnement spécifique et fluctuant tel que le système fluvio-estuarien de la Gironde (présence du bouchon vaseux et de forts courants des eaux).

Les temps de persistance obtenus (exprimés en «temps de demi-vie »: le temps nécessaire pour la perte de la moitié de la masse initiale) de trois espèces représentatives (peuplier, chêne, saule) des bassins versants en fonction de trois contextes hydrosédimentaires différents (pleine eau avec et sans courant, crème de vase) est toujours inférieur à l'année, compris majoritairement entre 1 et 4 mois selon les espèces considérées, plus ou moins résistantes. En outre, il ressort que le taux de dégradation est directement corrélé aux conditions environnementales que peut rencontrer la fraction végétale en transit dans l'estuaire (Figs. 6 et 7).
Dans des eaux de transition, certains travaux avaient suggéré l'influence des courants tidaux comme facteur abiotique déterminant la cinétique du processus de décomposition (Grout et al., 1997 ; Sangiorgio et al., 2008). Ceci est évident d'après nos résultats. En effet, en leur présence, la cinétique de dégradation est favorisée jusqu'à $30 \%$, à travers des processus d'abrasion et de fragmentation physique, comme observé par ailleurs (Lepori et al., 2005).

Au contraire, quand les apports foliaires entrent en contact avec les dépôts de vase, la cinétique du processus de dégradation diminue (jusqu'à $50 \%$ ). Diverses causes peuvent être évoquées: la réduction de l'activité microbienne aérobie due au caractère anoxique de la crème de vase (Naamane et al., 1999), l'absence d'abrasion physique provenant des courants tidaux entre la masse turbide (Sottolichio et al., 2011) et la réduction de stress physique.

Ces observations signifient que toute variation hydrodynamique ou morphologique en Gironde a des conséquences sur la persistance des feuilles et leur cinétique de dégradation (Fuentes-Cid et al., 2013).

Enfin, l'analyse des échelles de temps associées aux processus de dégradation du matériel végétal, obtenues dans ce travail, permet d'affirmer que le mécanisme d'apparition de Sar dans l'estuaire est relativement court (quelques mois) et qu'il démarre lors de la saison automnale. II ressort aussi l'influence décisive de la phase de transport sur la manière dont la matière 


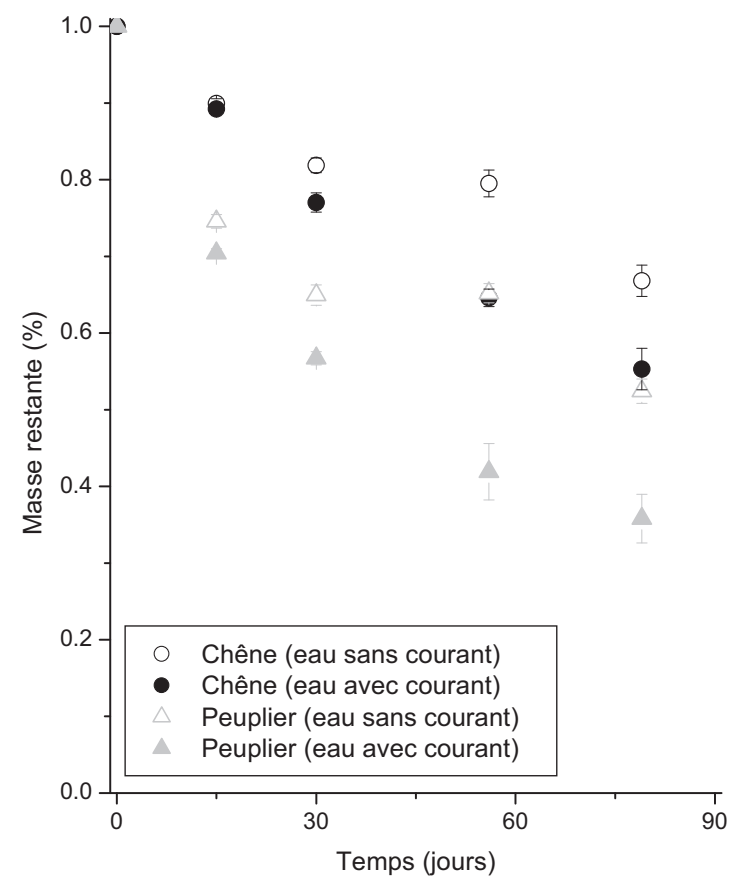

Fig. 6. Effet des courants tidaux sur la perte de masse au fil du temps, en fonction de deux espèces de feuilles (chêne et peuplier).

Fig. 6. Tidal currents effect on the loss weight through the time, according to the two species of leaves (oak and poplar).

organique foliaire évolue (cycles dépôt/ remise en suspension), notamment au vu de son rôle sur la durée et le mode de son transit à travers l'estuaire.

\subsection{Transport du Sar}

Le forçage hydrodynamique et hydrologique est décisif dans la dynamique de transport des débris végétaux dans l'estuaire.

Les débits fluviaux sont le vecteur majeur régissant les apports et le transport de matériel solide en milieu fluvio-estuarien et la quantité de débris végétaux apportée dans le système est proportionnelle à leur intensité. Dans l'estuaire de la Gironde, par exemple, la quantité estimée de débris végétaux transportés lors d'une crue peut représenter 5 fois la quantité transitant en conditions de débits fluviaux moyens (Fuentes-Cid, 2014).

Les mécanismes de descente et de re-distribution de matériel issu du bassin versant dans le lit des fleuves ou déposé sur leurs berges sont donc dominés, dans un premier temps, par le régime hydrologique fluvial, et, une fois arrivés dans les eaux estuariennes, par la combinaison des débits fluviaux et de la marée. Un suivi mensuel de l'évolution spatio-temporelle 




Fig. 7. Effet de dépôt des feuilles dans la crème de vase sur la perte de masse de feuilles de chêne. Fig. 7. Effect of leaves settling in the fluid-mud on the weight loss of oak leaves.

du matériel végétal allochtone dans l'estuaire central (Fuentes-Cid et al., 2014) a été mené sur trois années (2011-2012-2013). L'analyse de ce suivi montre que la dynamique de transport suit un comportement de type «flux-pulsé » : le facteur déclenchant étant une phase de crue hivernale, comme observé lors de la crue de début de l'année 2013, responsable de la présence potentiellement problématique des débris végétaux colmatant dans les eaux estuariennes $\left(0,03 \mathrm{~g} \cdot \mathrm{m}^{-3}\right.$ de fraction $>3 \mathrm{~mm}$ ) (cf. 2.3.c). Toutefois, les données montrent qu'il y a possibilité de blocage des débris végétaux dans l'estuaire (zone amont ou centrale) en fonction des débits. Si l'essentiel des débris feuillus parvient aux fleuves en période automnale et/ou hivernale, la tendance actuelle d'évolution des débits à la baisse, surtout depuis les deux dernières décennies (Fig. 8), plaide en faveur de phénomènes de dépôt dans les zones fluviales de la Garonne et de la Dordogne.

En zone fluvio-estuarienne, aucune accumulation notable de matériel végétal n'a été retrouvée dans les carottes effectuées dans cette section (Fuentes-Cid, 2014). Les conditions hydrosédimentologiques semblaient pourtant très favorables à ce processus, mais les apports de débris végétaux feuillus (estimés < $1 \%$ à $2 \%$ maximum des MES du bouchon vaseux) sont vraisemblablement dilués 


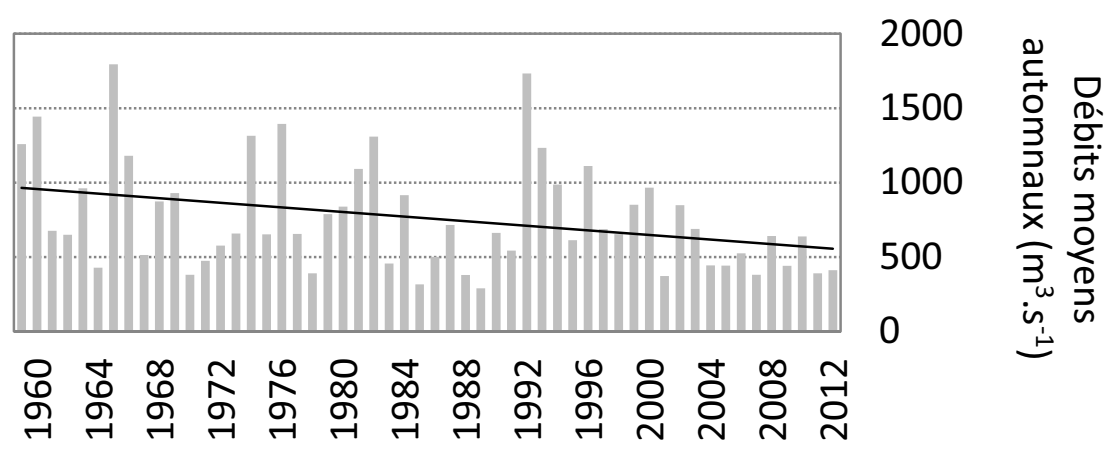

Fig. 8. Tendance évolutive des débits moyens automnaux (Garonne+Dordonge) de 1959 à 2012.

Fig. 8. Progressive trend of autumnal average freshwater discharge (Garonne+Dordogne) from 1959 to 2012.

dans la masse turbide du bouchon vaseux présente dans ces sections lors des faibles débits.

Par ailleurs, les courants tidaux contrôlent la resuspension des débris végétaux. Alors qu'en mortes-eaux, les eaux de fond sont systématiquement plus concentrées en débris végétaux que celles de surface, cette règle peut être altérée lors de vives-eaux. De plus, les fractions de taille $>3 \mathrm{~mm}$ (taille potentiellement problématique pour le CNPE) apparaissent dans la colonne d'eau, quand les vitesses de courant sont maximales lors de vives-eaux.

En résumé, le mécanisme de transport des débris végétaux dans l'estuaire varie selon trois contextes hydrologiques différents (Fig. 9).

a) Débits fluviaux faibles

Lorsque les débits fluviaux sont faibles $\left(<400 \mathrm{~m}^{3} \cdot \mathrm{s}^{-1}\right.$ : Garonne + Dordogne), pendant la période automne-hiver, les débris végétaux sont peu présents dans l'estuaire central. Dans la zone près du CNPE, les valeurs observées restent inférieures à 0,2 g.m ${ }^{3}$ (Fuentes-Cid et al., 2014).
Si la durée et l'intensité de l'étiage sont très prononcées, il peut exister du matériel feuillu accumulé dans les lits des cours d'eau non emporté par le faible courant, et conséquemment, le matériel végétal déposé sur les rives ou sur les plaines alluviales adjacentes au fleuve ne sera pas non plus amené au système aqueux.

Ainsi, peut-on suggérer l'existence d'une accumulation de matériel végétal fluvial dans la zone de confrontation amont des eaux fluviales et marines (sections fluvio-estuariennes). Ce stockage devrait être largement favorisé dans cette interface, de préférence aux autres zones soumises au régime tidal, car c'est la zone qui reçoit directement les apports de l'amont, la descente vers l'aval à partir de cette zone devenant spécialement lente dans ces conditions hydrologiques faibles. Ces secteurs de confrontation des masses d'eau présentent le plus souvent une turbidité très élevée avec l'apparition de crème de vase. Leur caractère anoxique ralentit la dégradation du matériel foliaire susceptible de s'y déposer, idée qui renforce le concept 



Fig. 9. Schéma conceptuel du transport des débris végétaux dans l'estuaire.

Fig. 9. Conceptual illustration of the vegetal debris transport in the estuary. 
d'accumulation possible tout au long des sections fluviales.

\section{b) Débits fluviaux moyens}

Quand les débits fluviaux, lors de la période automne/hiver, sont compris entre 400 et $1200 \mathrm{~m}^{3} . \mathrm{s}^{-1}$, la descente du matériel foliaire, dont celui temporairement déposé dans le lit des fleuves, est assez efficace jusqu'à l'arrivée dans la zone de balancement de marée. Les mesures de concentration en débris végétaux relevées par le suivi mensuel dans l'estuaire central (Fuentes-Cid et al., 2014) confirment cette arrivée des débris végétaux. Au niveau du PK 30 (à l'aval du Bec d'Ambés), la concentration de fraction végétale est détectable (généralement entre 0,1 et $1,6 \mathrm{~g} \cdot \mathrm{m}^{3}$ ) et d'un ordre de grandeur supérieur à celle mesurée $20 \mathrm{~km}$ en aval (aux environs du CNPE), où elle est souvent négligeable (entre 0,01 et 0,1 g. $\mathrm{m}^{3}$ ) (Fuentes-Cid et al., 2014). Ceci témoigne de la proximité des sources de débris végétaux en amont.

L'hypothèse, proposée pour expliquer ces faits, est l'interaction probable du matériel foliaire avec l'hydrologie et les matières en suspension (MES), de nature cohésive, dans l'estuaire amont. Une fois le matériel soumis aux courants tidaux, il connaît des cycles de dépôt et de remise en suspension, similaires à ceux des MES. La coexistence des débris végétaux et des MES peut alors compromettre la dynamique propre du matériel végétal, en rendant plus difficile sa remise en suspension. En conséquence, ce phénomène a pour effet de prolonger son temps de persistance dans le système.

Cette situation type "débits moyens " représente le cas de distribution des débris végétaux le plus fréquemment observé lors de cette étude (2011-2013).

c) Débits fluviaux forts à très forts

Les débits fluviaux forts ou très forts $\left(>1200 \mathrm{~m} \cdot \mathrm{s}^{-1}\right)$, en hiver ou début de printemps, permettent l'arrivée et la présence des débris végétaux dans l'estuaire central, voire aval. L'intensité des débits fluviaux régit strictement le temps de transit depuis l'amont et l'importance des concentrations de débris végétaux dans l'estuaire.

Le suivi mensuel a permis d'observer l'augmentation des concentrations en débris végétaux qu'une crue peut induire dans les eaux de l'estuaire central. Dans la zone près du CNPE, les concentrations ont augmenté de l'ordre de 10 fois par rapport aux données recueillies avec des débits fluviaux faibles. Contrairement au postulat de départ, cet accroissement est plus prononcé aux alentours du CNPE $(\times 10)$ que dans la zone de confluence de la Garonne et la Dordogne (×6) (Fuentes et al., 2014). L'explication en serait l'accroissement de l'efficacité du mécanisme de transport depuis l'amont jusqu'à l'estuaire central au moins. De manière "métaphorique ", une crue pourrait donc être considérée comme " un pont » qui lie les parties amont et aval de l'estuaire, permettant une évacuation en continu. En absence de crue, cette communication est bloquée, du fait du fonctionnement hydrodynamique de l'estuaire soumis à l'influence de la marée.

De par la comparaison des caractéristiques biogéochimiques des collectes avant et après la crue, le matériel végétal post-crue dans l'estuaire 
central témoigne d'un niveau de dégradation moindre (Fig. 5) avec des débris de taille supérieure à $3 \mathrm{~mm}$, c'est-àdire supérieure à la maille des tambours de filtration (Fuentes-Cid et al., 2014). Ces résultats confortent l'idée d'un transport relativement rapide et direct des débris végétaux depuis les bassins versants, sans étapes de dépôts estuariens intermédiaires.

\section{PHÉNOMÈNES DE COLMATAGE AU CNPE DU BLAYAIS}

\subsection{Dynamique de la présence des débris végétaux dans les tambours de filtration}

Les prises d'eau du CNPE servant à aspirer l'eau nécessaire pour satisfaire les besoins du circuit de refroidissement sont situées dans la colonne d'eau à environ trois mètres de profondeur. Habituellement, dans un contexte hydrologique moyen, l'eau dans cette zone présente une quantité de débris végétaux très faible, voire inexistante. Cependant, deux paramètres peuvent modifier la quantité des débris végétaux présents dans les eaux et leur qualité : une crue provenant des bassins versants, vecteur d'apport et de transport de ce matériel végétal, ou la remise en suspension des débris végétaux éventuellement déposés sur les fonds, phénomène favorisé principalement par les courants tidaux (cycle lunaire semi-diurne ou bimensuel) ou par l'action du vent.

Quant aux cycles de flot et jusant semi-diurnes auxquels les eaux estuariennes sont soumises quotidiennement, la figure 10 illustre les

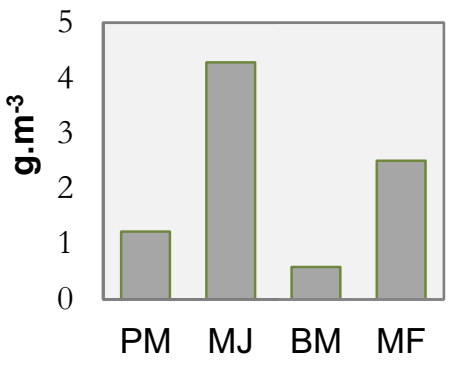

Fig. 10. Concentration (en poids sec) des débris végétaux prélevés dans les eaux de lavage des tambours lors d'un cycle de marée semi-diurne (PM : pleine mer; $\mathrm{MJ}$ : mi-jusant; $\mathrm{BM}$ : basse mer; MF: mi-flot). Données du 24 janvier 2011 ; coefficient de marée : 93.

Fig. 10. Vegetal debris concentration (dry mass) from the rotating drums washing water during a semidiurnal tide (PM: high tide; MJ: mid-ebb; BM: low tide; MF: mid-flood). Data of 24th January 2011; tidal coefficient: 93.

concentrations relevées sur les tambours de filtration : les quantités les plus élevées de débris végétaux sont mesurées à mi-jusant et à mi-flot, et les plus basses à pleine et basse mer. D'une part, cette tendance est similaire à celle des MES enregistrée par le réseau MAGEST (Etcheber et al., 2011), et, d'autre part, elle est cohérente avec l'occurrence des passages en grande vitesse (GV) des tambours filtrants dans la période comprise entre 3 et 1 heure avant la basse mer (Fig. 11).

La vitesse des courants de marée est soumise à des modulations par le cycle lunaire bimensuel, auquel se superpose le cycle de marée semidiurne. L'augmentation des coefficients de marée peut induire un accroissement direct (jusqu'à 4 fois supérieur) de la concentration de débris végétaux présents dans la colonne d'eau (Fuentes-Cid, 2014). 


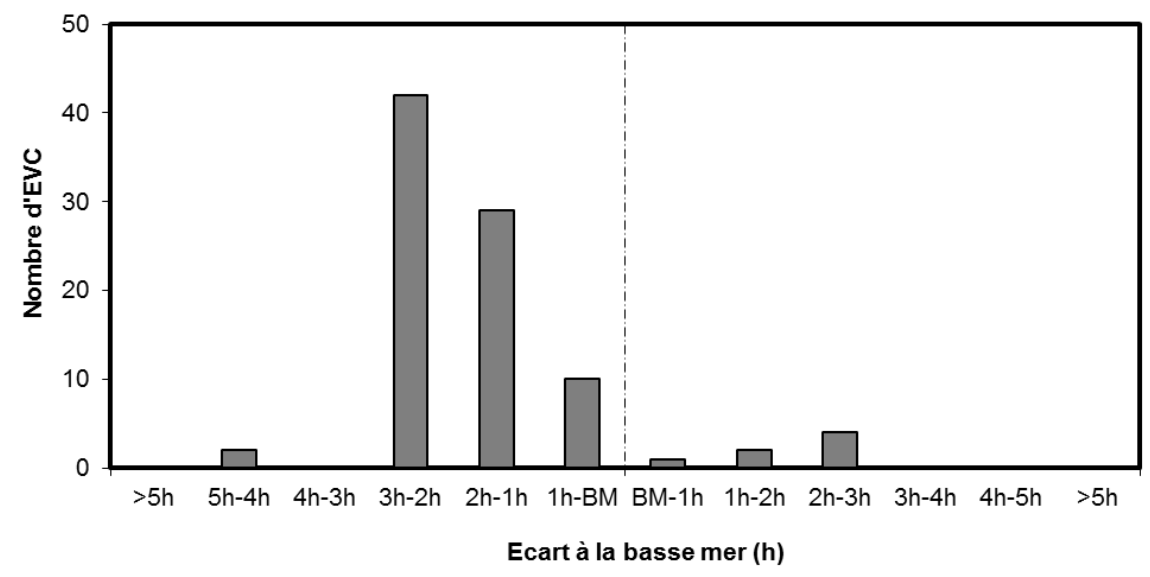

Fig. 11. Répartition des déclenchements de pompes dans le cycle de marée entre 1982 et 2012.

Fig. 11. Distribution of the pumps shut down through a semidiurnal tidal cycle from 1982 to 2012.

\subsection{Importance de la quantité} et de la qualité de la fraction végétale : suivi de l'évènement de colmatage de 2013 (arrêt de pompe)

La période à risque de colmatages de l'année 2013 (janvier-avril) a été analysée à travers la mise en relation des évènements de colmatage et les mesures de concentration de débris végétaux dans les eaux estuariennes, ainsi que dans les eaux de lavage des tambours de filtration du CNPE du Blayais.

L'enregistrement des passages en grande vitesse des tambours rotatifs de filtration est cohérent avec la présence accrue de débris végétaux détectés dans l'estuaire (fractions 1-3 mm et 3$5 \mathrm{~mm}$ ), dans les eaux de surface ou de fond (Fuentes-Cid et al., 2014). La figure 12 montre que la crue favorise la présence de débris végétaux dans l'estuaire central, qui entraîne des changements dans la vitesse de rotation des tambours de filtration du
CNPE (A), mais avec un effet retardé par rapport à la crue. En 2013, ce déphasage de temps a représenté près de deux mois, et celui-ci est cohérent avec, d'une part, la relation déjà évoquée dans les travaux de Fuentes-Cid et al. (2012) et, d'autre part, les résultats obtenus récemment (Fuentes-Cid, 2014) : plus la crue est forte, plus le temps d'arrivée du matériel végétal au CNPE est court (Fig. 13). Le contexte crue-colmatage de l'année 2013 constitue le décalage temporel observé (entre une crue et un colmatage au CNPE) le plus long (2 mois pour une crue de $4000 \mathrm{~m}^{3} \cdot \mathrm{s}^{-1}$ ), alors que le cas le plus rapide s'est produit lors de la crue de 2009 (17 jours pour une crue de $\left.6000 \mathrm{~m}^{3} \cdot \mathrm{s}^{-1}\right)$.

Les filtres rotatifs du CNPE accélèrent leur vitesse de rotation suite à l'impact de la crue (Fig. 12). Entre la période qui précède et qui suit l'augmentation des débits fluviaux, il existe un changement très prononcé de la vitesse de rotation, dite alors « grande 


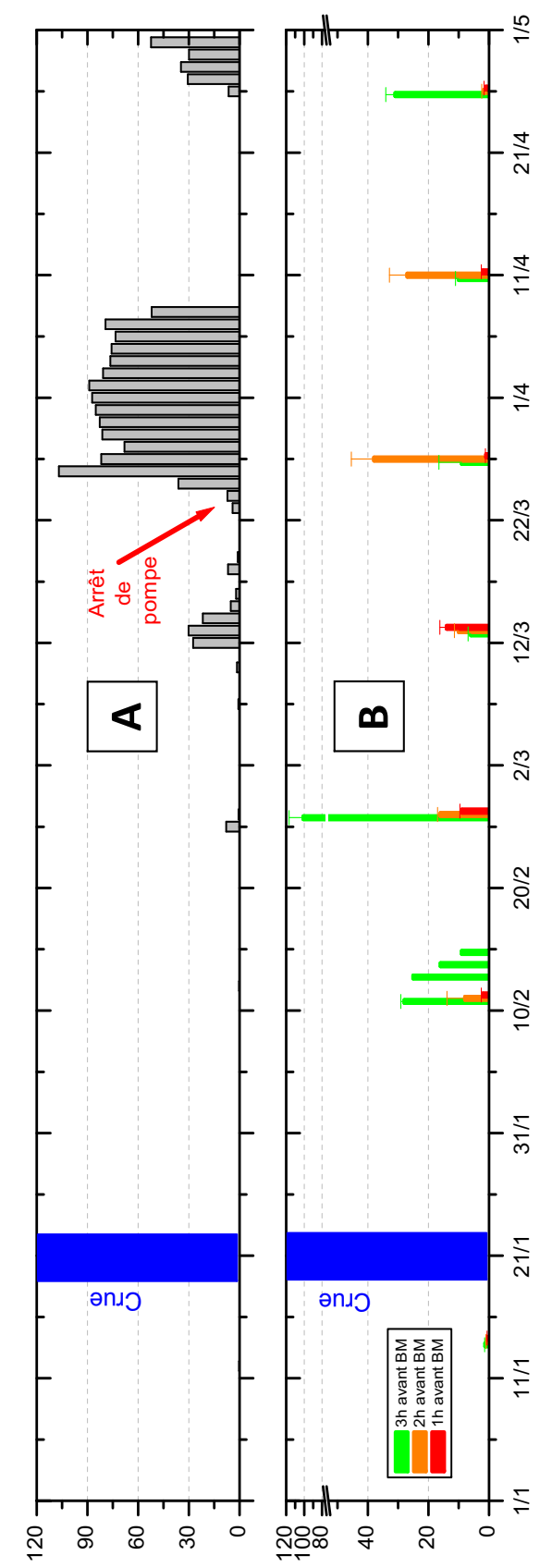

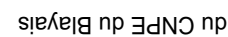
uo!̣eגו‼ әр sınoquet 8 sәр әбеме| әр хneә sә suep

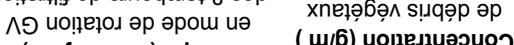


๘

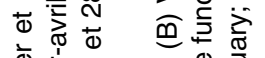

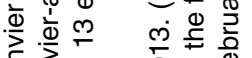

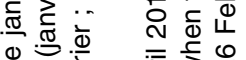
ब. ब 잉

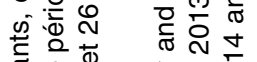

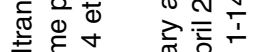
की 西

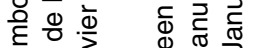
का के ट्ञ $\infty$ 흐 잉 舟 人)

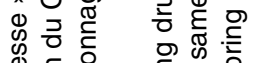
这. 등 잉

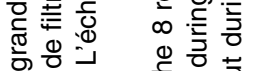
ه 헝 을

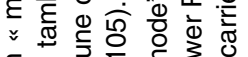
ธ긍

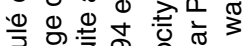

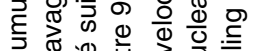

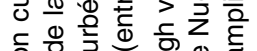

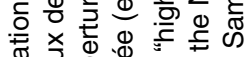
需



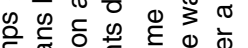
है

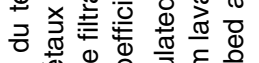

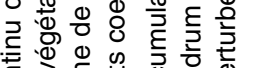





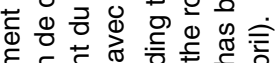

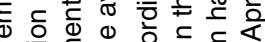

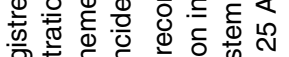

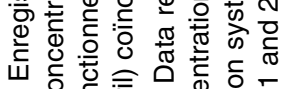

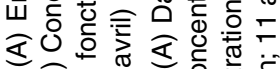

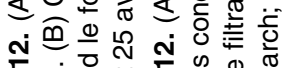








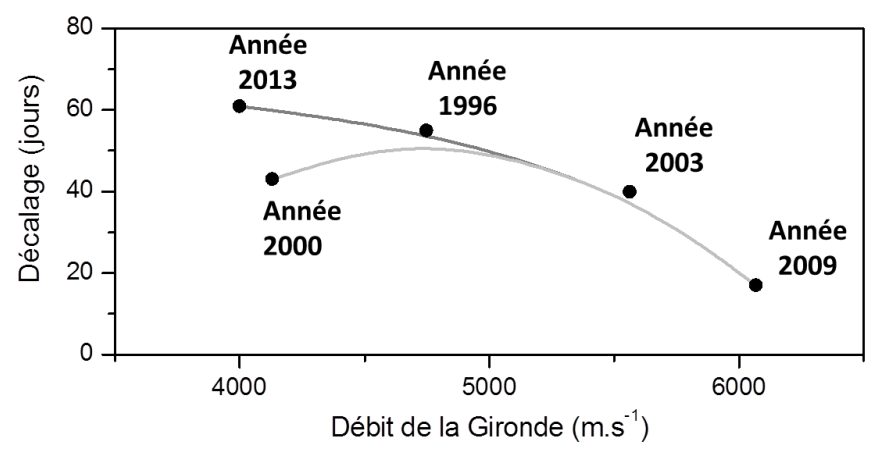

Fig. 13. Temps (jour) entre l'occurrence d'une crue en Gironde et un évènement de colmatage dans le système de filtration du CNPE du Blayais en fonction des débits. Les courbes donnent l'enveloppe de répartition des décalages temporels entre la date du pic de crue et l'observation de colmatage. Extrait de Fuentes-Cid (2014).

Fig. 13. Time (day) between a flood in the Gironde watershed and the blockage intake event in the filtration system of the Blayais Nuclear Power Plant as a function of water discharges. The curves cover the temporal gap between the flood peak and the blockage intake event. Extracted from Fuentes-Cid (2014).

vitesse (GV) ». Cette figure 12 montre aussi un arrêt de pompe survenu au CNPE du Blayais le 23 mars. À ce moment-là, la quantité maximale de débris végétaux mesurés dans les eaux de lavage des filtres rotatifs (Fig. 12) n'a pas révélé, contrairement au postulat de départ, des valeurs aussi élevées que celles observées un mois avant (le 26 février). Pour cette raison, il faut évoquer la qualité du matériel végétal feuillu comme paramètre régulant les phénomènes de colmatage. En effet, l'efficacité du processus d'évacuation des débris végétaux à travers le système de nettoyage est dépendante de l'état structurel des débris et de leur pouvoir colmatant.

Sur 4 des 7 campagnes menées en 2013, la concentration maximale des débris végétaux dans les eaux de lavage des tambours de filtration est constatée 3 heures avant la basse mer
(Fig. 12). Cette tendance, considérée comme la tendance "normale », rejoint le comportement vu à l'échelle du cycle de marée semi-diurne: les concentrations maximales de MES (Etcheber et al., 2011) et/ou de débris végétaux dans la colonne d'eau sont observées à mi- marée (Fig. 10). Par contre, trois exceptions à la tendance normale ont été notées et ces trois cas d'«altération de tendance » ont été observés simultanément à de fortes perturbations dans les tambours (passages GV) : mi-mars, fin mars et miavril (Fig. 12). Ce fait s'explique par la saturation évidente du tambour filtrant face à l'arrivée du matériel végétal colmatant. Cette fraction " collée » à la maille filtrante n'est pas évacuée, de façon efficace, du tambour au fur et à mesure de son arrivée. En conséquence, ces débris végétaux restent un temps plus ou moins long en contact avec les tambours de filtration 
avant d'être évacués. Ce fait peut affecter le passage des eaux vers les pompes du système de refroidissement. L'augmentation des concentrations de débris végétaux dans les eaux de lavage du tambour filtrant est alors décalée de 1 ou 2 heures par rapport aux entrées, comme il a été constaté.

L'examen de l'historique du moment d'occurrence des passages GV (Fig. 12) montre que le pic de passages GV de mi-mars, période où l'arrêt de pompes a eu lieu, coïncide avec l'altération de tendance repérée au même moment (Fig. 12).

Cette analyse met donc en évidence la sensibilité des tambours filtrants, face à la quantité et à la qualité de matériel végétal présent dans les eaux. Cependant, la biomasse, seule, des débris végétaux qui s'accumulent sur la surface filtrante des tambours n'est pas suffisante pour expliquer les colmatages comme le montrent les concentrations des débris végétaux au CNPE mises en regard avec l'historique des vitesses de rotation des tambours. En cohérence avec le mécanisme de transport décrit précédemment, les colmatages sont dus à des débris de matériel foliaire déplacés par "flux pulsé », dont l'état de dégradation est relativement moins avancé que celui des débris présents habituellement dans la zone des prises d'eau, et de taille $(>3 \mathrm{~mm}$ ) susceptible de colmater les tambours de filtration (maille : $3 \mathrm{~mm}$ ).

\section{Conclusions et perspectives}

Ce travail valide l'hypothèse que la dynamique d'apports et de transport des débris végétaux dans l'estuaire de la Gironde suit un comportement de type «flux pulsé », fortement tributaire des débits fluviaux. Les zones considérées comme émettrices de débris végétaux doivent être circonscrites majoritairement dans les sections moyennes des bassins versants de la Garonne et de la Dordogne, zones caractérisées par des boisements fluviaux denses et des sections sensibles à la montée des débits fluviaux.

Les projections hydro-climatologiques pour la Région Aquitaine confirment une tendance à la baisse des débits fluviaux, déjà observée lors des deux dernières décennies (Etcheber et al., 2013) (Fig. 8). Si les débits fluviaux, vecteurs majeurs d'apport et de transport de matériel foliaire depuis leurs sources, tendent à diminuer lors de la saison automnale, la probabilité que les litières à l'automne restent dans les zones ripariennes et dans des bras déconnectés du cours d'eau principal sera plus élevée. Au lieu d'apports diffus sur plusieurs semaines, ce matériel serait alors remobilisé en période de crues et lorsque le lit majeur est inondé. La conséquence serait alors l'existence de crues charriant des flux de débris végétaux encore plus prononcés, capables de transiter directement jusqu'à l'estuaire central. Cette évolution possible doit être prise en compte, car elle conduirait à des risques accrus pour le fonctionnement du CNPE, même à des débits inférieurs à $4000 \mathrm{~m}^{3} \cdot \mathrm{s}^{-1}$.

De plus, la baisse des débits a une incidence sur la remontée du bouchon vaseux vers les sections fluviales. Les tendances actuelles y indiquent une occurrence plus longue des fortes 
concentrations de MES. Les implications sur le transport des débris végétaux devraient donc favoriser un blocage de matériel végétal dans les sections amont. Ceci pourrait constituer une zone de piégeage de ce matériel, qui se dégraderait alors de façon lente, s'il y a enfouissement dans de la crème de vase, et qui pourrait être ensuite remobilisé à la fin des périodes d'étiage, lors d'augmentation notable des débits fluviaux.

Trois types de perspectives sont envisagés pour continuer à améliorer la compréhension de la dynamique des débris végétaux dans le système fluvio-estuarien de la Gironde et les impacts sur le CNPE.

La prise en compte du réseau de retenues et de barrage (essentiellement sur la Dordogne) permettrait d'estimer leurs impacts sur la biomasse et la temporalité des concentrations en débris végétaux. Cette approche permettrait d'étayer l'hypothèse du transport «flux-pulsé ». Actuellement, les accumulations des débris végétaux en amont des barrages restent largement inconnues, et l'estimation de leur contribution (à travers des campagnes de carottage, par exemple) au flux total susceptible d'être transféré vers l'aval permettrait de mieux relativiser son importance, lors des phases de crue plus spécialement. De plus, cerner les contributions relatives en débris végétaux des deux fleuves Garonne et Dordogne pourrait être entrepris, soit directement à travers la mise au point des méthodologies classiques d'échantillonnage permettant leur quantification, soit à travers l'application d'un marqueur/traceur capable de spécifier l'origine du matériel qui arrive aux tambours filtrants du CNPE.

La seconde approche concerne la prédiction des arrivées massives de Sar au CNPE. L'utilisation par des approches statistiques, des échelles de temps et d'espace associées à la dynamique du matériel végétal et du bouchon vaseux, de l'intensité des débits fluviaux, ainsi que les données relatives aux perturbations des tambours filtrant, permettrait la mise en place de campagnes de prélèvements en pleine eau pour améliorer le système de prévision de risque de colmatage.

La troisième approche concerne la modélisation numérique du transport du Sar à l'échelle de l'estuaire pour simuler les déplacements des débris végétaux. Pour cela, il est nécessaire de connaître les caractéristiques physiques des débris afin d'en déduire leur dynamique de transport: vitesse de chute, de remise en suspension ... Ce type d'outil offrirait la possibilité de tester les hypothèses de transport et de cibler des zones d'accumulation (si elles existent), avec à terme, un modèle prenant en compte l'origine, le transport et la dégradation des débris végétaux.

\section{RÉFÉRENCES BIBLIOGRAPHIQUES}

Abelho M., 2001. From Litterfall to Breakdown in Streams: A Review. The Scientific World 1 : 656-680.

Batalla R.J. \& Vericat D., 2009. Hydrological and sediment transport dynamics of flushing flows: implications for management in large Mediterranean Rivers. 
River Research and Applications 25 (3) : 297-314.

Chauvet E., 1987. Changes in the chemical composition of alder, poplar and willow leaves during decomposition in a river. Hydrobiologia 148 (1) : 35-44.

David V., Sautour B., Chardy P. \& Leconte M., 2005. Long-term changes of the zooplankton variability in a turbid environment: The Gironde estuary (France). Estuarine, Coastal and Shelf Science 64 (2-3) : 171-184.

Etcheber H., Taillez A., Abril G., Garnier J., Servais P., Moatar F. \& Commarieu M.-V., 2007. Particulate organic carbon in the estuarine turbidity maxima of the Gironde, Loire and Seine estuaries: origin and lability. Hydrobiologia 588 (1) : 245-259.

Etcheber H., Schmidt S., Sottolichio A., Maneux E., Chabaux G., Escalier J.M. Wennekes H., Derriennic H., Schmeltz M., Quéméner L., Repecaud M., Woerther P. \& Castaing P., 2011. Monitoring water quality in estuarine environments: lessons from the MAGEST monitoring program in the Gironde fluvial-estuarine system. Hydrol. Earth Syst. Sci. 15 (3) : 831-840.

Etcheber H., Coynel A., Sauquet E. \& Coupry B., 2013. Disponibilité des eaux de surface. In Le treut $H$., Ed., Les impacts du changement climatique en Aquitaine. Presses Universitaires de Bordeaux, 265-274.

Fuentes-Cid A., 2014. Étude pluridisciplinaire d'une perturbation industrielle dans l'estuaire de la Gironde : implications du transport et de la dynamique de dégradation des débris végétaux sur le fonctionnement de la source froide du CNPE du Blayais, Thèse Univ. Bordeaux.

Fuentes-Cid A., Etcheber H., Schmidt S., Sottolichio A., De-Oliveira E., Oggian G. \& Derriennic H., 2012. Dynamique spatio-temporelle du matériel végétal grossier dans l'estuaire de la Gironde, Paralia, 281-288.

Fuentes-Cid A., Chauvet E., Etcheber H., De-Oliveira E., Sottolichio A. \& Schmidt S., 2013. Leaf litter degradation in highly turbid transitional waters: preliminary results from litter-bag experiments in the Gironde Estuary. Geodinamica Acta, 1-7.

Fuentes-Cid A., Etcheber H., Schmidt S., Abril G., De-Oliveira E., Lepage M. \& Sottolichio A., 2014. Dynamics of coarse particulate matter in the turbidity maximum zone of the Gironde Estuary. Comptes Rendus Geoscience, 346 : 28-36.

Gessner M.O. \& Chauvet E., 2002. A case for using litter breakdown to assess functional stream integrity. Ecological Applications 12 (2) : 498-510.

Gessner M.O., Chauvet E. \& Dobson M., 1999. A perspective on leaf litter breakdown in streams. Oikos 85 (2): 377-384.

Girardin M., Lepage, M. \& Gonthier P., 2004. Colmatage des tambours filtrants du Centre Nucléaire de Production Électrique du Blayais : Campagne de mesures dans l'estuaire de la Gironde (Campagnes GIRSAR), Rapport Cemagref- Étude $\mathrm{n}^{\circ} 85$ - Departement Gestion des Milieux Aquatiques - Unité Ressources Aquatiques Continentales, $19 \mathrm{p}$.

Girardin M., Lepage M. \& Gonthier P., 2006. Colmatage des tambours filtrants du Centre Nucléaire de Production Électrique du Blayais : Campagnes de mesures dans l'estuaire de la Gironde (Campagnes GIRSAR 2006), Rapport Etude ${ }^{\circ} 106$ - Département Gestion des Milieux Aquatiques - Unité Ressources Aquatiques Continentales, $18 \mathrm{p}$.

Gómez C.M., Pérez-Blanco C.D. \& Batalla R.J., 2013. Tradeoffs in river 
restoration: Flushing flows vs. hydropower generation in the Lower Ebro River, Spain. Journal of Hydrology, in press.

Grout J., Levings C. \& Richardson J., 1997. Decomposition rates of purple loosestrife (Lythrum salicaria) and Lyngbyei's sedge (Carex lyngbyei) in the Fraser River estuary. Estuaries 20 (1) : 96-102.

Guibert A. \& Lebarh R., 2008. Colmatage des tambours filtrants du Centre Nucléaire de Production Électrique du Blayais: Campagne de mesures dans l'estuaire de la Gironde (Campagne GIRSAR 2008), Rapport - Département Gestion de Milieux Aquatiques - Unité Ressources Aquatiques Continentales, $18 \mathrm{p}$.

Guibert A., Ballion B. \& Bouju V., 2009. Colmatage des tambours filtrants du Centre Nucléaire de Production Électrique du Blayais: Campagne de mesures dans l'estuaire de la Gironde, Rapport Cemagref. Département Gestion des Milieux Aquatiques - Unité Ressources Aquatiques Continentales, $14 \mathrm{p}$.

Irigoien X. \& Castel J., 1997. Light Limitation and Distribution of Chlorophyll Pigments in a Highly Turbid Estuary: the Gironde (SW France). Estuarine, Coastal and Shelf Science 44 (4) : 507-517.

Issa R., 2010. Projet « SASHA »P10W90: contrat de projet - Version A. Rapport EDF R\&D, $53 \mathrm{p}$.

Lecerf A., Risnoveanu G., Popescu C., Gessner M.O. \& Chauvet E., 2007. Decomposition of diverse litter mixtures in streams. Ecology 88 (1) : 219-227.

Lepage M., Gonthier P. \& Girardin M., 2003. Colmatage des tambours filtrants du CNPE du Blayais. Campagnes de mesures dans l'estuaire de la Gironde (Campagnes GIRSAR). Rapport Cemagref-Étude ${ }^{\circ} 81$ - Département Gestion des Milieux Aquatiques - Unité Ressources Aquatiques Continentales, $18 \mathrm{p}$.
Lepori F., Palm D. \& Malmqvist B., 2005. Effects of stream restoration on ecosystem functioning: detritus retentiveness and decomposition. Journal of Applied Ecology 42 (2) : 228-238.

Lopes M.L., Martins P., Ricardo F., Rodrigues A.M. \& Quintino V., 2011. In situ experimental decomposition studies in estuaries: A comparison of Phragmites australis and Fucus vesiculosus. Estuarine, Coastal and Shelf Science 92 (4) : 573-580.

Mateo M.A. \& Romero J., 1996. Evaluating seagrass leaf litter decomposition: an experimental comparison between litter-bag and oxygen-uptake methods. Journal of Experimental Marine Biology and Ecology 202 (2) : 97-106.

Montemayor D.I., Addino M., Fanjul E., Escapa M., Alvarez M.F., Botto F. \& Iribarne O.O., 2011. Effect of dominant Spartina species on salt marsh detritus production in SW Atlantic estuaries. Journal of Sea Research 66 (2) : 104110.

Naamane B., Chergui H. \& Pattee E., 1999. The breakdown of leaves of poplar and holm oak in three Moroccan streams: effect of burial in the sediment. Ann. Limnol.- Int. J. Lim. 35 (04) : 263-275.

Palau A., Batalla R., Rosico E., Meseguer A. \& Vericat D., 2004. Management of water level and design of flushing floods for environmental river maintenance downstream of the Riba-roja reservoir (lower Ebro River, NE Spain), HYDRO 2004 - A New Era for Hydropower. Porto, Portugal, 18-20 October 2004.

Quintino V., Sangiorgio F., Ricardo F., Mamede R., Pires A., Freitas R., Rodrigues A.M. \& Basset A., 2009. In situ experimental study of reed leaf decomposition along a full salinity gradient. Estuarine, Coastal and Shelf Science 85 (3) : 497-506. 
Sangiorgio F., Basset A., Pinna M., Sabetta L., Abbiati M., Ponti M., Minocci M., Orfanidis S., Nicolaidou A., Moncheva S., Trayanova A., Georgescu L., Dragan S., Beqiraj S., Koutsoubas D., EvagelopouIos A. \& Reizopoulou S., 2008. Environmental factors affecting Phragmites australis litter decomposition in Mediterranean and Black Sea transitional waters. Aquatic Conservation: Marine and Freshwater Ecosystems 18 (S1) : S16-S26.

Savoye N., David V., Morisseau F., Etcheber H., Abril G., Billy I., Charlier K., Oggian G., Derriennic H. \& Sautour B., 2012. Origin and composition of particulate organic matter in a macrotidal turbid estuary: The Gironde Estuary, France. Estuarine, Coastal and Shelf Science 108 (0) : 16-28.

Sottolichio A., Castaing P., Etcheber H., Maneux E., Schmeltz M. \& Schmidt S.,
2011. Observations of suspended sediment dynamics in a highly turbid macrotidal estuary, derived from continuous monitoring. Journal of Coastal Research SI 64.

Travade F., 2002. CNPE du Blayais. Analyse des débris colmatant les tambours filtrants, Rapport EDF R\&D LNHE HP-76/ 02/031/A.

Travade F. \& Guerin C., 2009. Colmatage des stations de pompage du CNPE du Blayais par les débris végétaux. Études in situ réalisées à la suite des évènements de 2003. Analyse des facteurs propices aux incidents (1996-2003), Rapport EDF R\&D LNHE HP-76-200900383-FR, $63 \mathrm{p}$.

WANO, 2008. Nouvelles instructions de WANO, Inside WANO. The magazine of the World Association of Nuclear Operators 16 (1) : 14-15. 